\title{
Enhancement of Fluoresceinyl Cypridina Luciferin Analog Chemiluminescence by Human Serum Albumin for Singlet Oxygen Detection
}

\author{
Jing Zhou ${ }^{1}$, Da Xing ${ }^{\star 1}$ and Qun Chen ${ }^{1,2}$ \\ ${ }^{1}$ MOE Key Laboratory of Laser Life Science and Institute of Laser Life Science, \\ South China Normal University, Guangzhou, Peoples Republic of China \\ ${ }^{2}$ Department of Radiation Oncology University of Colorado at Denver Health Sciences Center, Aurora, CO
}

Received 2 December 2005; accepted 12 April 2006; published online 18 April 2006 DOI: 10.1562/2005-12-02-RA-744

\begin{abstract}
Fluoresceinyl cypridina luciferin analog (FCLA) is a chemiluminescence $(\mathrm{CL})$ probe for detecting reactive oxygen species (ROS). Its efficiency for detecting singlet oxygen $\left({ }^{1} \mathrm{O}_{2}\right)$ can be significantly enhanced in the presence of human serum albumin (HSA). This phenomenon may apply to important applications for both research and clinical testing, because of the broad presence of HSA in the human system. In the current study the mechanism of the FCLA-HSA CL system is studied by means of direct CL measurement and spectroscopy techniques. Our results show that FCLA can combine with HSA via a single binding site to form a complex. The CL efficiency of the system is largely governed by an intersystem energy transfer between the two components upon interaction with ${ }^{1} \mathrm{O}_{2}$. The $\mathrm{CL}$ production reaches maximum in a synergetic manner when equal amounts of FCLA and HSA are present simultaneously, but production is less at other ratios. The results also show that the combination of FCLA with HSA does not significantly alter the ROS selectivity of FCLA. In conclusion, our study shows that FCLA and HSA can combine and form a complex with higher CL efficiency. This provides us a new approach in designing $C L$ techniques for studying ROS.
\end{abstract}

\section{INTRODUCTION}

Singlet oxygen $\left({ }^{1} \mathrm{O}_{2}\right)$ is an excited state of molecular oxygen and one of the most important reactive oxygen species (ROS). It plays an important role in biological systems and is responsible for many types of tissue damage and an extraordinary array of diseases $(1,2)$. Accordingly, detection of the generation and distribution of ${ }^{1} \mathrm{O}_{2}$ in biologic systems has great significance for both basic and clinical research. At present there are several methods for ${ }^{1} \mathrm{O}_{2}$ detection, including electron spin resonance, stopped flow spectrophotometry, ultra-weak chemiluminescence (CL), and direct ${ }^{1} \mathrm{O}_{2}$ luminescence (3-7). CL has been reported to be a sensitive and convenient method to detect the existence of ${ }^{1} \mathrm{O}_{2}$ in complicated biologic systems (8-10). The basic principle of this

*Corresponding author email: xingda@scnu.edu.cn (Da Xing) (c) 2006 American Society for Photobiology 0031-8655/06 method is that the free radical with an elevated energy level can react with a specific CL probe and initiate a series of chemical reactions that releases additional chemical energy. This results in the structurally altered probe molecule reaching an excited state. The subsequent de-excitation of the probe emits photons (CL), typically in the visible wavelength range. Compared with the other methods, the intensity and temporal profile of CL are relatively easy to record with conventional optical systems, such as a photon multiplier tube (PMT) and/or charge-coupled device (CCD).

Fluoresceinyl cypridina luciferin analog (FCLA) is a wellestablished CL probe for ROS $(9,10)$. Its significant characteristics important for biological applications include that the CL photons have the longest wavelength among the known chemiluminescent substances in the category (11) and its optimum environmental $\mathrm{pH}$ is within the typical physiological range (12).

Human serum albumin (HSA) is a major protein component of blood plasma. This protein contains a single intrinsic tryptophan and has internal dimensions favorable for the Förster type of energy transfer (13). HSA can easily bind to many agents and transport them in the bloodstream $(14,15)$. It is confirmed that, in a normal physiological environment, HSA is in a favorable structural conformation for binding with a great majority of drugs (16). In addition, HSA is a known ${ }^{1} \mathrm{O}_{2}$ quencher and can readily react with ${ }^{1} \mathrm{O}_{2}$ (17-19) and generate $450 \mathrm{~nm} \mathrm{CL}$ (although very weakly) (19). The CL is estimated to be emitted from a triplet state with the carbonyl group produced by the reaction between proteins and ${ }^{1} \mathrm{O}_{2}(19,20)$.

We have previous reported that the efficiency of FCLA CL could be enhanced in the presence of HSA $(21,22)$. The mechanism of the enhancement and its potential applications were not discussed. The objective of the current study is to study the CL mechanism of FCLA-HSA system.

\section{MATERIALS AND METHODS}

Reagents. FCLA (free acid [3,7-dihydro-6-[4-[2-[ $\left[\mathrm{N}^{\prime}-(5\right.$-fluoresceinyl) thioureido]ethoxy] phenyl]-2-methylimidazo[1,2-a]pyrazin-3-one], Tokyo Kasei Kogyo Co., Tokyo, Japan) was dissolved in double-distilled water at a concentration of $100 \mu \mathrm{mol} \mathrm{L} \mathrm{L}^{-1}$ and stored at $-80^{\circ} \mathrm{C}$ until needed. A stock solution of protoporphyrin IX disodium salt (PpIX) (Aldrich Chem. Co., Milwaukee, WI) was prepared according to the manufacturer's instructions to a concentration of $200 \mu \mathrm{mol} \mathrm{L} \mathrm{L}^{-1}$. The stock was stored in the dark at $4^{\circ} \mathrm{C}$ until needed. HSA (purity, $>96 \%$; Sigma, St. Louis, MO) was

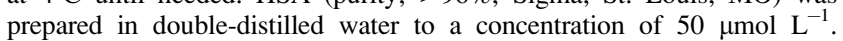




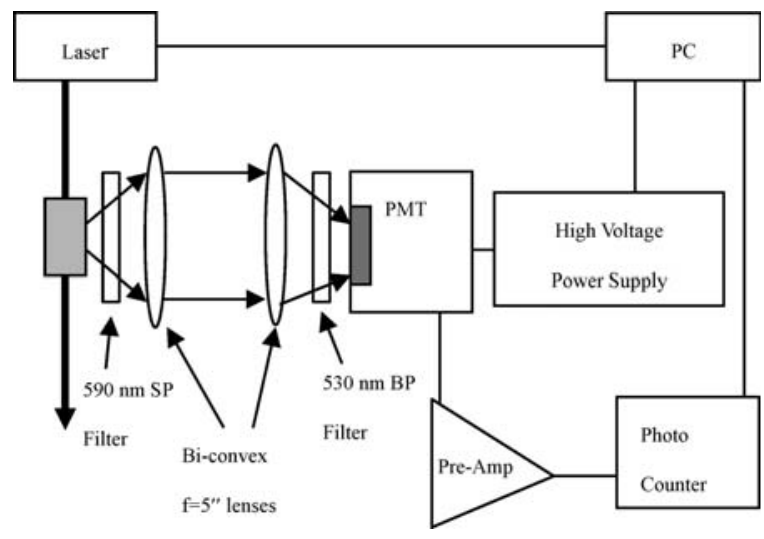

Figure 1. Schematic of the experimental setup used for CL measurements.

Sodium azide $\left(\mathrm{NaN}_{3}\right)$, hydrogen peroxide $(30 \%)$ and sodium hypochlorite were purchased from the Guangzhou Chemical Reagent Company.

Singlet oxygen production. In the current study singlet oxygen was produced via two distinctively different methods: a photosensitization reaction and a direct chemical reaction.

In the photosensitization reaction ${ }^{1} \mathrm{O}_{2}$ was generated by light irradiation of photosensitizer PpIX. The irradiation light source was a custom-built $635 \mathrm{~nm}, 200 \mathrm{~mW}$ diode laser (diode controller: LDC2000; temperature controller: TEC2000; Thorlab, Newton, NJ).

In the direct chemical reaction ${ }^{1} \mathrm{O}_{2}$ was produced from the $\mathrm{H}_{2} \mathrm{O}_{2} / \mathrm{NaOCl}$ chemical reaction. The concentration of $\mathrm{H}_{2} \mathrm{O}_{2}$ and $\mathrm{NaOCl}$ was $200 \mathrm{mmol}$ $\mathrm{L}^{-1}$ and $3 \mathrm{~mol} \mathrm{~L}^{-1}$, respectively. The solution was titrated to $\mathrm{pH}$ 7.4. To compare the alter of HSA CL and FCLA CL, the concentrations of FCLA and HSA were set at $0.01 \mu \mathrm{mol} \mathrm{L}{ }^{-1}$ and $5 \mu \mathrm{mol} \mathrm{L}^{-1}$. To control the timing of ${ }^{1} \mathrm{O}_{2}$ production, $\mathrm{NaOCl}$ was injected into the $\mathrm{H}_{2} \mathrm{O}_{2}$ in a glass cuvette $\left(0.5 \times 1 \times 4 \mathrm{~cm}^{3}\right)$ while CL was recorded.

Experimental setup for CL detection. The schematic for CL measurement (and that of light irradiation for the photosensitization reaction) is summarized in Figure 1. In the current study FCLA CL was measured in real-time using a PMT (model 952; Perkin Elmer Optoelectronics Instruments) operating in single-photon counting mode. The CL measurement setup was identical for both studies, although there was no laser irradiation in the direct chemical reaction study. All CL measurements were made at room temperature and the data were sampled every $0.2 \mathrm{~s}$ (sampling rate, $5 \mathrm{~Hz} \mathrm{~s}^{-1}$ ).

For the photosensitization study a $590 \mathrm{~nm}$ short-pass (SP) and a $530 \mathrm{~nm}$ band-pass (BP) filter were used in front of the PMT to minimize the scattered excitation light $(635 \mathrm{~nm})$ but allow the $530 \mathrm{~nm} \mathrm{CL}$ photons to reach the detector. The optical axis of the irradiation light was set at a $90^{\circ}$ angle to the detector, to further improve the signal-to-noise ratio. For all photosensitization experiments the samples were irradiated uniformly with a light fluence rate of $25.5 \mathrm{~mW} \mathrm{~cm}$. For comparison of the $\mathrm{CL}$ from HSA and FCLA in the $\mathrm{H}_{2} \mathrm{O}_{2} / \mathrm{NaOCl}$ chemical reaction, CL was measured before and after FCLA and HSA were mixed, using either a $455 \mathrm{~nm}$ (for HSA) or a $527 \mathrm{~nm}$ (for FCLA) BP filter set between the sample and the PMT for signal discrimination.

Experimental procedures. To simplify the investigation the study was performed in a cell-free environment. After HSA and FCLA were mixed at different ratios according to the experimental protocols ([HSA]/ [FCLA]:0.25 to 3 with [FCLA] $=1$ or $5 \mu \mathrm{mol} \mathrm{L}^{-1}$ was added to the solution. In the current study the order of chemical addition was very important to avoid HSA interaction with the photosensitizer. The $\mathrm{pH}$ of the samples was adjusted to a standard level of 7.4. The prepared sample was immediately irradiated according to the protocol for the photosensitization reaction.

The ${ }^{1} \mathrm{O}_{2}$ production rate was varied by changing the concentration of PpIX $\left(0.2-2 \mu \mathrm{mol} \mathrm{L}^{-1}\right)$. CL from $5 \mu \mathrm{mol} \mathrm{L}{ }^{-1}$ FCLA and $5 \mu \mathrm{mol} \mathrm{L}^{-1}$ FCLA mixed with $5 \mu \mathrm{mol} \mathrm{L}{ }^{-1}$ HSA were measured from different concentrations of ${ }^{1} \mathrm{O}_{2}$.

To identify the nature of the enhanced CL when FCLA is combined with HSA, total CL production from FCLA alone $\left(5 \mu \mathrm{mol} \mathrm{L}^{-1}\right)$ and the FCLAHSA mixture $\left(5 \mu \mathrm{mol} \mathrm{L}{ }^{-1}\right.$ each) was measured by continuously irradiating the samples until the signal diminished to the background level.

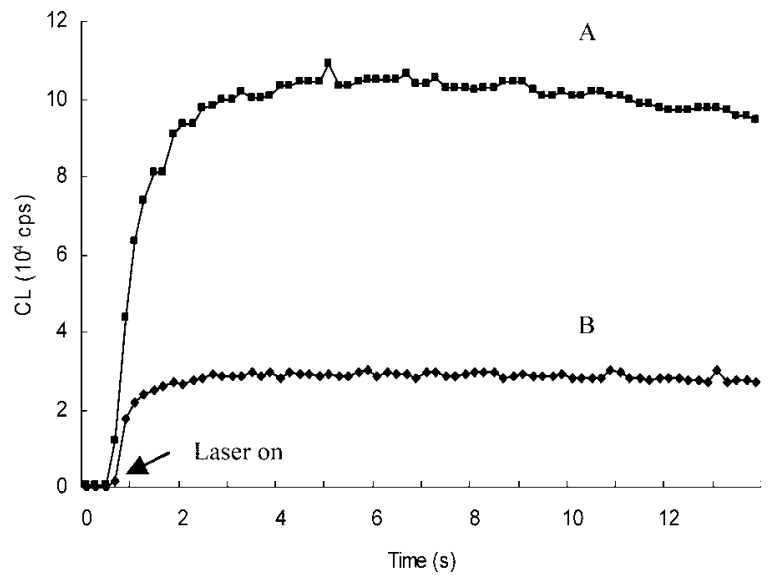

Figure 2. Comparison of real-time $\mathrm{CL}$ intensity in photosensitization reaction with $5 \mu \mathrm{mol} \mathrm{L}{ }^{-1}$ FCLA, $2 \mu \mathrm{mol} \mathrm{L}{ }^{-1}$ PpIX and a $635 \mathrm{~nm}, 25.5 \mathrm{~mW}$ $\mathrm{cm}^{-2}$ laser fluence rate in the presence (A) and absence (B) of $5 \mu \mathrm{mol} \mathrm{L}-1$ HSA.

To verify the ROS selectivity of FCLA and FCLA-HSA a known ${ }^{1} \mathrm{O}_{2}$ quencher, $\mathrm{NaN}_{3}\left(1 \mathrm{mmol} \mathrm{L}^{-1}\right)$ was added to the solution before the light measurement. The concentrations of FCLA and HSA were both $5 \mu \mathrm{mol} \mathrm{L}{ }^{-1}$.

Spectroscopic measurements. To identify potential structural change and/or binding of the probe and HSA, absorption spectra of FCLA and HSA alone and of the FCLA-HSA mixture were recorded with a Lambda 35 UV/VIS spectrometer (Perkin Elmer). The fluorescence and CL spectra of the samples were recorded with a LS 55 luminescence spectrometer (Perkin Elmer). For the FCLA-quenching of HSA fluorescence, various concentrations of FCLA and a fixed concentration of HSA were mixed to make a total volume of $600 \mu \mathrm{L}$ in a standard quartz cuvette. The final protein concentration was $2.5 \mu \mathrm{mol} \mathrm{L} \mathrm{L}^{-1}$, and the molar ratios of FCLA to protein varied from 0 to 4 . The fluorescence spectra were recorded with 279 $\mathrm{nm}$ excitation. All experiments were performed at room temperature.

\section{RESULTS}

\section{CL measurements of FCLA with and without HSA during the photosensitization reaction}

Photosensitization reaction, similar to photodynamic therapy, provides a very well-controlled ${ }^{1} \mathrm{O}_{2}$ source. With light irradiation there was a noticeable luminescence ( $c a 400 \mathrm{cps}$ at laser fluence rate of $25.5 \mathrm{~mW} \mathrm{~cm}^{-2}$ ) due to scattered irradiation light reaching the detector. This background luminescence was nearly identical for samples containing water only, water with HSA, water with PpIX, water with HSA and PpIX only. For samples containing FCLA but no light irradiation we observed a small but consistent luminescence signal above the dark-current counting of the optical system (Fig. 2). The background luminescence without light irradiation was likely due to the FCLA self-oxidation.

After subtraction of the background luminescence and scattered irradiation light FCLA CL was observed from all samples containing FCLA and PpIX, regardless of the presence or absence of HSA. The CL intensity was consistently stronger in samples from the FCLA-HSA mixture, compared with samples from FCLA alone. Figure 2 shows a representative comparison of the real-time $\mathrm{CL}$ intensities.

Figure 3 shows the CL intensities for various HSA/FCLA ratios at two fixed FCLA concentrations. For each FCLA concentration the largest $\mathrm{CL}$ intensity was observed in samples with equal molar 


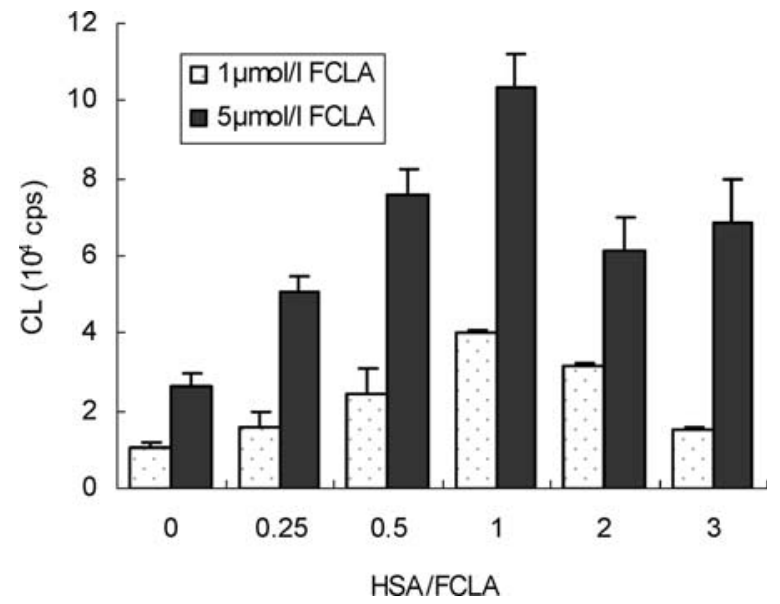

Figure 3. Comparison of $C L$ intensities from various HSA/FCLA concentration ratios with $5 \mu \mathrm{mol} \mathrm{L} \mathrm{L}^{-1}$ or $1 \mu \mathrm{mol} \mathrm{L}{ }^{-1}$ FCLA, $2 \mu \mathrm{mol} \mathrm{L} \mathrm{L}^{-1}$ PpIX and $25.5 \mathrm{~mW} \mathrm{~cm}^{-2}$ fluence rate. The largest CL intensity was observed in samples with equal molar concentrations of FCLA and HSA. Data are presented as mean $\pm \mathrm{SD}$ of at least three separate experiments.

concentrations of FCLA and HSA and was nearly four times that in FCLA alone.

Figure 4 shows the CL intensity comparisons of FCLA and FCLA-HSA for different PpIX concentrations. The CL enhancement by HSA was consistently observed for all photosensitizer concentrations investigated.

The CL was also measured during continuous irradiation until the signal diminished to the background level. The results show that, given identical experimental protocols, the integrated luminescence signal from the FCLA-HSA mixture was seven times that from FCLA alone (Fig. 5).

Without FCLA, CL from HSA alone during the photosensitization reaction was extremely weak and practically undetectable with the current experiment setup. It is clear that the CL enhancement observed for the FCLA and HSA mixture was a synergetic effect rather than a simple summation.

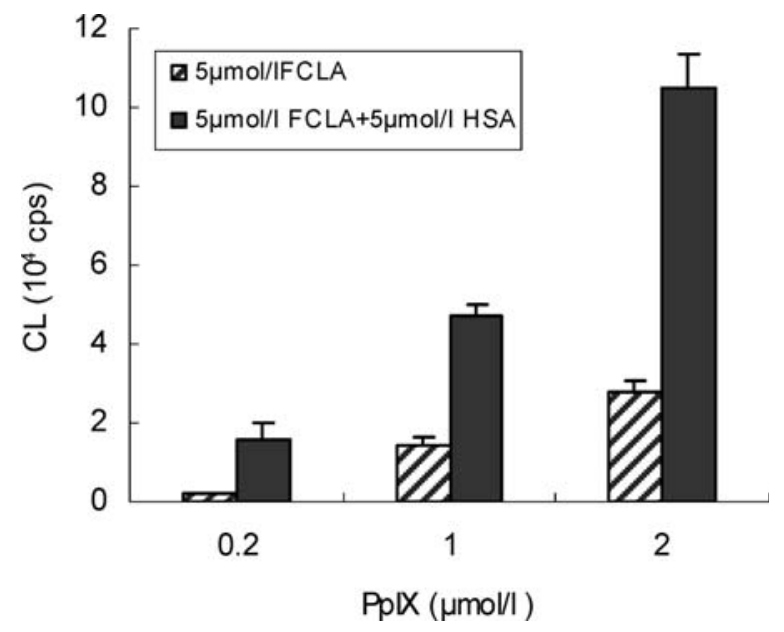

Figure 4. Comparison of CL intensities from different concentrations of PpIX with $5 \mu \mathrm{mol} \mathrm{L} \mathrm{L}^{-1}$ FCLA in the absence of and in the presence of $5 \mu \mathrm{mol} \mathrm{L}{ }^{-1}$ HSA. Data are presented as mean \pm SD of at least three separate experiments.

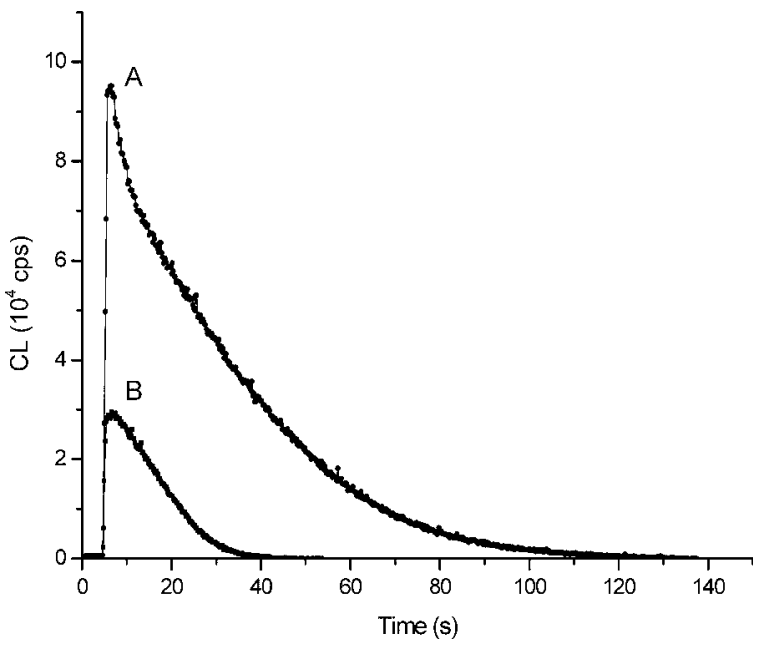

Figure 5. CL of $5 \mu \mathrm{mol} \mathrm{L}{ }^{-1}$ FCLA in the presence (A) and absence (B) of $5 \mu \mathrm{mol} \mathrm{L}{ }^{-1}$ HSA with continuous irradiation until the signal diminished to the background level .

\section{Effect of ${ }^{1} \mathrm{O}_{2}$ quencher $\mathrm{NaN}_{3}$ on FCLA CL and FCLA-HSA CL in photosensitization reaction}

To investigate the origins of FCLA CL and FCLA-HSA CL in the current study, CL from photosensitization reaction was evaluated with and without the ${ }^{1} \mathrm{O}_{2}$ quencher $\mathrm{NaN}_{3}$. As shown in Figure 6 FCLA CL was quenched 64\% and FCLA-HSA CL was quenched $79 \%$ by $\mathrm{NaN}_{3}$.

\section{Stoichiometry of FCLA-HSA interaction and binding}

To analyze the characteristics of the FCLA-HSA binding, the stoichiometry of HSA fluorescence quenching by FCLA was investigated. The results show that, when mixed with different concentrations of FCLA, the fluorescence of HSA at $337 \mathrm{~nm}$ was quenched proportionally in comparison to its control value (Fig. 7). Aside from the self-quenching mechanism, HSA fluorescence may also be quenched by several other mechanisms, such as dynamic and static quenching. Considering that the volume of FCLA in the experiments was far smaller than that of HSA, the dilution

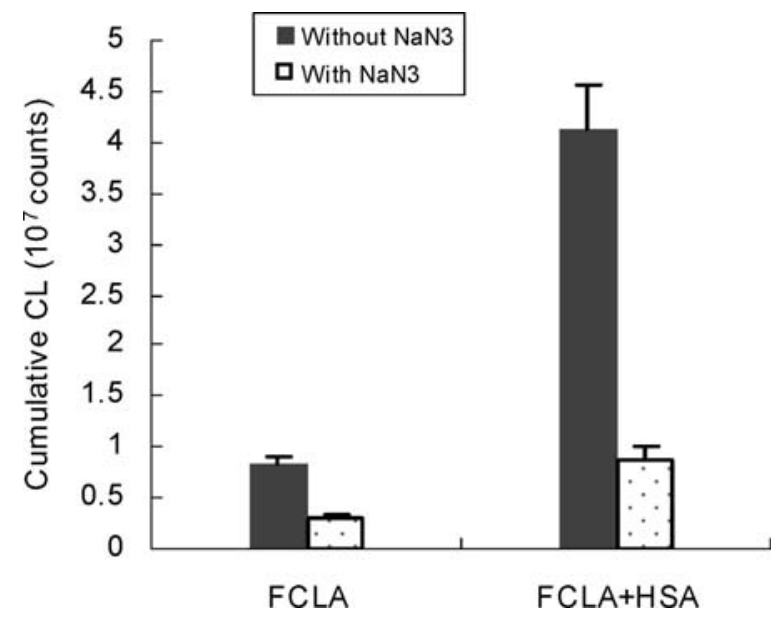

Figure 6. Effect of $1 \mu \mathrm{mol} \mathrm{L} \mathrm{L}^{-1} \mathrm{NaN}_{3}$ on FCLA CL and FCLA-HSA CL in the photosensitization reaction. Data are presented as mean $\pm \mathrm{SD}$ of at least three separate experiments. 


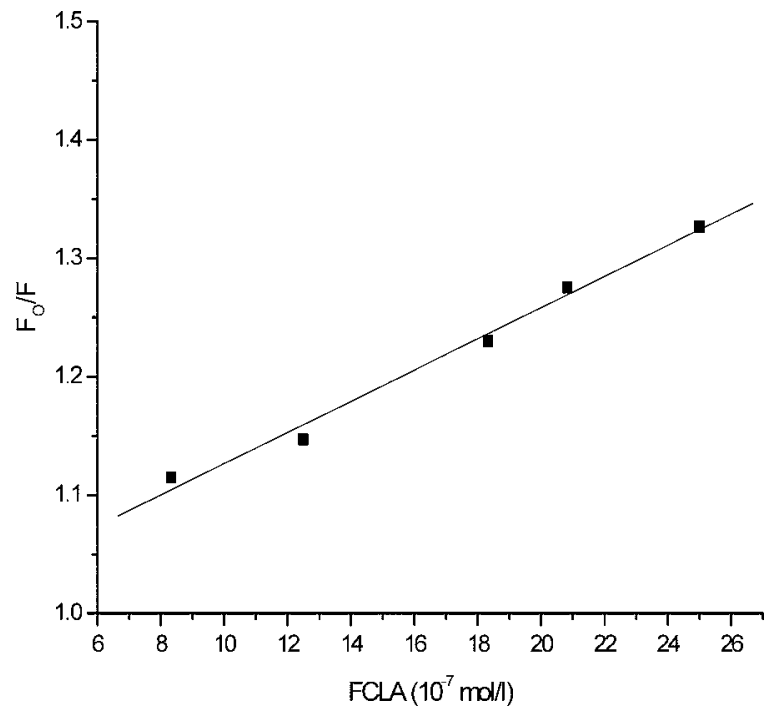

Figure 7. The curve of the relationship between $F_{0} / F$ and the concentration of FCLA.

effect of FCLA was thus negligible. Dynamic quenching is caused by physical collision among molecules and can be described by the Stern-Volmer equation (23)

$$
\frac{F_{0}}{F}-1=\mathrm{k}_{\mathrm{q}} \cdot \tau_{0}[\mathrm{Q}]=\mathrm{K}_{\mathrm{D}}[\mathrm{Q}]
$$

where $\mathrm{F}$ and $\mathrm{F}_{0}$ are the relative fluorescence intensities of HSA in the presence and absence of quencher $\mathrm{Q}$ (FCLA), respectively. $\mathrm{k}_{\mathrm{q}}$ is the quenching constant. $\tau_{0}$ is the excited-state lifetime in the absence of Q (approximately $10^{-8}$ s for macromolecules) (24). $K_{D}$ is the Stern-Volmer constant. The quenching constant, $\mathrm{k}_{\mathrm{q}}$, for the FCLA-HSA system was calculated using the data shown in Figure 7. With the Stern-Volmer equation kq was found to be $1.31 \times 10^{13}$ $\mathrm{L} \mathrm{mol}{ }^{-1} \mathrm{~s}^{-1}$. This value is much larger than that of the limiting diffusion rate constant of typical biomolecules $\left(2 \times 10^{10} \mathrm{~L} \mathrm{~mol}^{-1}\right.$ $\mathrm{s}^{-1}$ ), so dynamic quenching is not likely to be a primary reason in

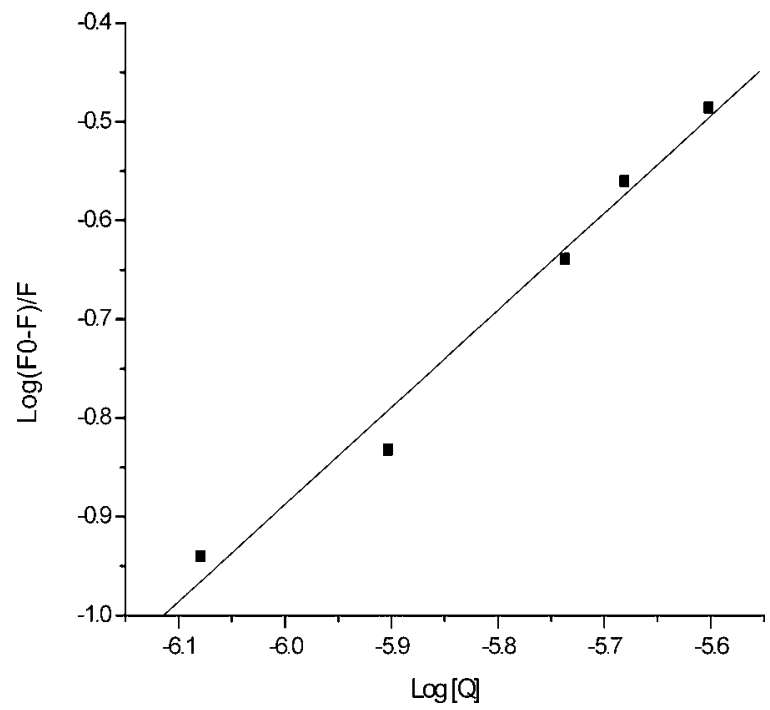

Figure 8. Logarithmic plot of fluorescence quenching of HSA treated with different FCLA concentrations. $\lambda \mathrm{ex}=279 \mathrm{~nm}$.

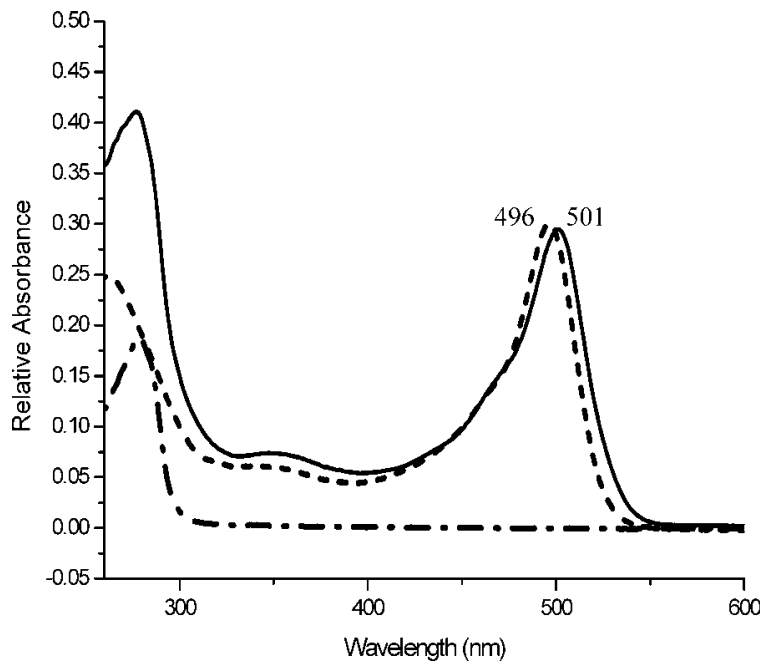

Figure 9. Absorption spectra of $5 \mu \mathrm{mol} \mathrm{L} \mathrm{L}^{-1}$ FCLA (dashed line), $5 \mu \mathrm{mol}$ $\mathrm{L}^{-1} \mathrm{HSA}$ (boxed line) and $5 \mu \mathrm{mol} \mathrm{L}{ }^{-1}$ FCLA mixed with $5 \mu \mathrm{mol} \mathrm{L}{ }^{-1} \mathrm{HSA}$ (solid line).

the quenching of HSA fluorescence $(25,26)$. Static quenching can result from the formation of a ground-state complex that is nonfluorescent or weakly fluorescent. According to Jiang et al. (27), if molecule Q has $n$ binding sites on protein HSA the relative protein fluorescence intensities $\mathrm{F}$ and $\mathrm{F}_{0}$ (in the presence and absence of $\mathrm{Q}$, respectively); the concentrations of $\mathrm{Q}$ in the reaction can be described as follows:

$$
\log \frac{F_{0}-F}{F}=\log \mathrm{Ka}+\operatorname{nlog}[\mathrm{Q}]
$$

In this study Q was regarded as probe FCLA. When the ratio of the concentration of probe to protein is less than or equal to $1: 1$, $\log \frac{F_{0}-F}{F}$ is highly linear to $\log [\mathrm{Q}](\mathrm{R}=0.99 ; P=0.001)$ (Figure 8). The slope of the linear regression (the number of binding sites) was found to be 0.98. This suggests that FCLA has only one binding site on HSA.

\section{Absorption and CL spectra of FCLA in the absence and the presence of HSA}

Figure 9 shows representative absorption spectra of FCLA, HSA and their mixture at a 1:1 concentration ratio. With HSA alone there was a distinctive peak at $279 \mathrm{~nm}$, whereas the major absorption peak of FCLA was at $496 \mathrm{~nm}$. When FCLA was mixed with HSA the absorption spectrum of the compound consisted of the two corresponding absorption peaks, with one important difference. Although the absorption peak wavelength of HSA at 279 nm did not change, the one corresponding to the FCLA had shifted $5 \mathrm{~nm}$ (from $496 \mathrm{~nm}$ to $501 \mathrm{~nm}$ ) when FCLA was mixed with HSA. The wavelength shift was also observed in the corresponding CL spectrum. As shown in Figure 10 the peak CL wavelength shifted from $522 \mathrm{~nm}$ of FCLA alone to $526 \mathrm{~nm}$ when FCLA was mixed with HSA.

\section{Excited-state intermolecular energy transfer in FCLA-HSA complexes}

To explore the mechanism of CL enhancement for the FCLAHSA complex, the fluorescence spectra of FCLA, HSA and their complex were further examined. Figure 11 shows the excited-state 


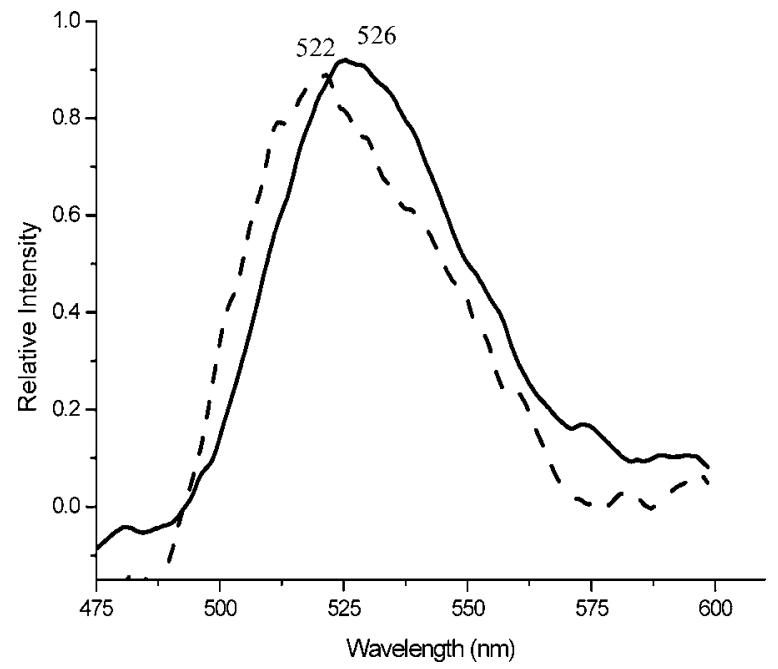

Figure 10. CL spectra of $5 \mu \mathrm{mol} \mathrm{L}{ }^{-1}$ FCLA in the absence (dashed line) and the presence (solid line) of $5 \mu \mathrm{mol} \mathrm{L}^{-1} \mathrm{HSA}$.

intermolecular energy transfer spectra in FCLA-HSA complexes. The binding of FCLA to HSA caused an efficient quenching of the HSA fluorescence. Simultaneously, the FCLA fluorescence intensity was enhanced. As shown in Figure 12, in the $\mathrm{H}_{2} \mathrm{O}_{2} / \mathrm{NaOCl}$ reaction there was an observable HSA CL at $450 \mathrm{~nm}$ when HSA alone reacted with ${ }^{1} \mathrm{O}_{2}$ (the background luminescence of FCLA self-oxidation was close to the dark-current counting of the optical system and was ignored). When FCLA was mixed with HSA the HSA CL at $450 \mathrm{~nm}$ was reduced and the FCLA CL at $526 \mathrm{~nm}$ was enhanced. The data clearly indicate that there is an intermolecular energy transfer from HSA at its excited state to FCLA in the FCLA-HSA complex.

\section{DISCUSSION}

We have previously reported that the efficiency of FCLA CL production is highly improved in the presence of HSA $(21,22)$. When FCLA and HSA are combined for detection of ${ }^{1} \mathrm{O}_{2}$ the resultant total $\mathrm{CL}$ is synergetic rather than a case of simple summation. Considering that HSA is a natural protein that is present in all parts of a human body and that CL has a broad range of potential applications, understanding the mechanism of this CL enhancement phenomenon becomes critical. The current study utilizes direct CL measurement and spectroscopy techniques to investigate the phenomenon and to analyze the underlying mechanism.

In the current study ${ }^{1} \mathrm{O}_{2}$ was produced from the following different systems: chemical reaction between $\mathrm{H}_{2} \mathrm{O}_{2}$ and $\mathrm{NaOCl}$ and a photosensitization reaction similar to that in photodynamic therapy. Although the ${ }^{1} \mathrm{O}_{2}$ source was different and the concentrations of ${ }^{1} \mathrm{O}_{2}$ were different (Fig. 4), the FCLA CL was enhanced by HSA in both cases, suggesting the enhancement was source independent. In a well-controlled photosensitization reaction with the ${ }^{1} \mathrm{O}_{2}$ quencher $\mathrm{NaN}_{3}$, CL was quenched $64 \%$ from FCLA alone; the value changed to $79 \%$ when HSA was added (Fig. 6). This clearly indicates that the addition of HSA did not hamper the specificity of the ROS probe, if not improve it.

The CL was measured with a fixed concentration of FCLA mixed with various concentrations of HSA in the photosensitization reaction. When the HSA-FCLA concentration ratio was $1: 1$, the largest CL intensity (close to 4 times that from FCLA alone)

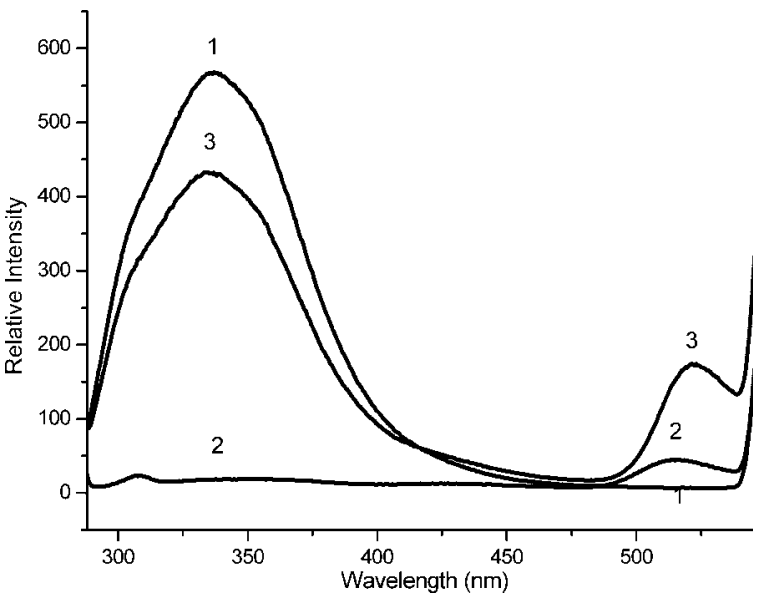

Figure 11. Fluorescence spectra of $2.5 \mu \mathrm{mol} \mathrm{L}^{-1} \mathrm{HSA}$ in the absence (1) and the presence (3) of $2.5 \mu \mathrm{mol} \mathrm{L} \mathrm{L}^{-1}$ FCLA. Curve 2 represents the fluorescence spectrum of $2.5 \mu \mathrm{mol} \mathrm{L}{ }^{-1}$ FCLA alone. $\lambda \mathrm{ex}=279 \mathrm{~nm}$.

was obtained (Fig. 3). The enhancement was found to be true both in the intensity at a given moment during an irradiation and in the overall integrated CL values. With further increase of the HSA concentration, the CL enhancement was less efficient (Fig. 3). This can be explained as follows. HSA alone is a known ${ }^{1} \mathrm{O}_{2}$ quencher. When excess HSA is present it directly reacts with and quenches ${ }^{1} \mathrm{O}_{2}$ to produce a protein carbonyl group. The elevated energy state of the protein carbonyl groups typically goes through nonradiative decay with low quantum yield in photon production (18). This results in a decreased amount of ${ }^{1} \mathrm{O}_{2}$ available for interaction with FCLA to produce CL.

The enhancement mechanism was further investigated on the basis of spectroscopy results. Evaluation of the stoichiometry of the HSA fluorescence quenching by FCLA revealed that FCLA can combine with HSA and that FCLA has only one binding site on HSA. This finding is confirmed by the finding that maximum CL enhancement was achieved in samples with equal amounts of HSA and FCLA. When FCLA was combined with HSA the peak FCLA absorption and CL wavelengths red-shifted by 5 and $4 \mathrm{~nm}$, respectively.

When combined with FCLA, it is likely that the ${ }^{1} \mathrm{O}_{2}$ quencher HSA can, at its elevated energy state, pass its energy via excitedstate intermolecular energy transfer to FCLA. Comparison of the fluorescence spectra for HSA, FCLA and the FCLA-HSA complex

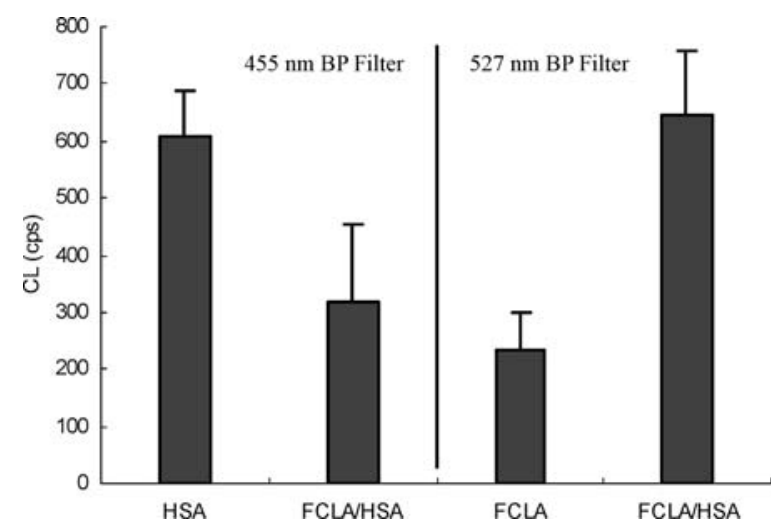

Figure 12. Comparison of HSA CL with FCLA CL before and after FCLA was mixed with $\mathrm{HSA}$ in the $\mathrm{H}_{2} \mathrm{O}_{2} / \mathrm{NaClO}$ solution. Data are presented as mean $\pm \mathrm{SD}$ of at least three separate experiments. 
(Fig. 11) and for HSA CL and FCLA CL (Fig. 12) clearly revealed that Förster energy transfer occurred in the FCLA-HSA complex. Because it is a very efficient ${ }^{1} \mathrm{O}_{2}$ quencher, HSA is easily excited by ${ }^{1} \mathrm{O}_{2}$. After it combines with FCLA the HSA protein carbonyl group (which is the product of reaction between HSA and ${ }^{1} \mathrm{O}_{2}$ ) transfers its elevated energy to excite FCLA. Therefore, there may be two luminescence processes by FCLA. First is the true CL process involving FCLA reaction with ${ }^{1} \mathrm{O}_{2}$ :

$$
\mathrm{FCLA}+{ }^{1} \mathrm{O}_{2} \rightarrow \text { FCLA }=\mathrm{O} * \rightarrow \text { FCLA }=\mathrm{O}+\mathrm{h} v
$$

This is an irreversible process in which the chemical structure of FCLA is altered; FCLA thus cannot be involved in future CL production. Second is the luminescence from the radiative transition of the excited FCLA due to the energy transfer from HSA. This is a reversible process in which FCLA molecules can recycle in photon production.

Given same amount of photosensitizer and irradiation fluence rate, CL was measured from samples containing FCLA alone and the FCLA-HSA mixture until the signals were reduced to background level (Fig. 5). In comparison, the value from FCLAHSA was a magnitude higher than that of FCLA alone. Fresh air/ oxygen was injected into the solvent after a certain period of irradiation during the measurement and we found little or no increase in CL (data not shown). This indicated that the oxygen consumption did not affect the singlet oxygen produced with the fixed concentration of PpIX. The result clearly demonstrates that radiative transition of the excited FCLA contributes greatly to the CL collected during the measurement. This is because FCLA CL is a nonreversible process: if there was no radiative transition due to direct excitation of FCLA by energy transferred from HSA, the total integrated CL from the two experiments would be similar.

The spectra results show that the peak wavelengths of fluorescence and the CL spectra of FCLA alone are $516 \mathrm{~nm}$ (Fig. 11) and $522 \mathrm{~nm}$, respectively. There is a $6 \mathrm{~nm}$ difference. When FCLA combines with HSA its fluorescence peak wavelength changes to $522 \mathrm{~nm}$ and the corresponding CL peak wavelength is $526 \mathrm{~nm}$. This is further evidence that the spectrum of the complex is a combination of FCLA CL and excited FCLA luminescence mediated by energy transfer from HSA.

Although there is no direct proof from the current study there are other potential mechanisms by which HSA can enhance the FCLA CL. This may include but not be limited to the following. Analysis of the FCLA CL mechanism reveals that the optimum reaction environment for FCLA CL is similar to that of the typical range found in physiological reactions. During the FCLA CL process there is an important intramolecular proton transfer via $\mathrm{H}_{2} \mathrm{O}$ assistance (28). HSA is a typical amphoteric molecular and is able to get and release a proton. When HSA is combined with FCLA it is likely that the presence of HSA favors the reaction between FCLA and ${ }^{1} \mathrm{O}_{2}$. The excitement of FCLA due to HSA energy transfer may also increase the chance of its CL reaction with ${ }^{1} \mathrm{O}_{2}$. Last but not least the presence of HSA may improve the quantum yield of photons via radiative de-excitation after the excited FCLA reacted with ${ }^{1} \mathrm{O}_{2}$, because of changes in microenvironment around the molecules.

In conclusion, the mechanism by which HSA enhances FCLA CL has been examined for the first time. The results demonstrate that the FCLA-HSA complex is a more efficient method for producing $\mathrm{CL}$ in ${ }^{1} \mathrm{O}_{2}$ detection, compared with FCLA alone. The enhancement in the photon production is due to an energy transfer process from HSA reacting with ${ }^{1} \mathrm{O}_{2}$ to excite FCLA.
Acknowledgements-This research is supported by the National Natural Science Foundation of China (grants 60378043 and 30470494), the Natural Science Foundation of Guangdong Province (grants 015012, 04010394 and 2004B10401011) and the National Institutes of Health (grant PO1-43892).

\section{REFERENCES}

1. Harman, D. (1984) Free radical and the origination, evolution, and present status of the free radical theory of aging. In Free Radicals in Molecular Biology, Aging, and Disease (Edited by D. Armstrong and R. S. Sohal), pp. 1-12. Raven Press, New York.

2. Harman, D. (1986) Free radical theory of aging: Role of free radicals in the origination and evolution of life, aging, and disease processes. In Free Radicals, Aging, and Degenerative Diseases (Edited by J. E. Johnson et al.), pp. 3-51. Alan R. Liss Inc., New York.

3. Tarpey, M. M. and I. Fridovich (2001) Methods of detection of vascular reactive species nitric oxide, superoxide, hydrogen peroxide, and peroxynitrite. Circ. Res. 89, 224-236.

4. Niedre, M. J., A. J. Secord, M. S. Patterson and B. C. Wilson (2003) In vitro tests of the validity of singlet oxygen luminescence measurements as a dose metric in photodynamic therapy. Cancer Res. 63, 7986-7994.

5. Niedre, M. J., C. S. Yu, M. S. Patterson and B. C. Wilson (2005) Singlet oxygen luminescence as an in vivo photodynamic therapy dose metric: Validation in normal mouse skin with topical amino-levulinic acid. Br. J. Cancer. 92, 298-304.

6. Baker, A. and J. R. Kanofsky (1991) Direct observation of singlet oxygen phosphorescence at $1270 \mathrm{~nm}$ from L1210 leukemia cells exposed to polyporphyrin and light. Arch. Biochem. Biophys. 286, 70-75.

7. Andersen, L. K., Z. Gao, P. R. Ogilby, L. Poulsen and I. Zebger (2002) A singlet oxygen image with 2.5 micrometer resolution. J. Phys. Chem. A. 106, 8488-8490.

8. Murphy, M. E. and H. Sies (1990) Chemiluminescence: Approaches to detecting and determining reactive oxygen species. Anal. Proc. 27, 217-219.

9. He, Y., D. Xing, G. Yan and K. Ueda (2002) FCLA chemiluminescence from sonodynamic action in vitro and in vivo. Cancer Lett. 182, 141-145.

10. Qin, Y. F., D. Xing, S. M. Luo, J. Zhou, X. Y. Zhong and Q. Chen (2005) Feasibility of using fluoresceinyl cypridina luciferin analog in a novel chemiluminescence method for real time photodynamic therapy dosimetry. Photochem. Photobiol. 81, 1534-1538.

11. Shimada, M., S. Kawamoto, Y. Nakatsuka and M. Watanabe (1993) Localization of superoxide anion in the red tide alga Chattonella antiqua. J. Histohem. Cytochem. 41, 507-511.

12. Oosthuizen, M. M. J., M. E. Engelbrecht, H. Lambrechts, D. Greyling and R. D. Levy (1997) The effect of $\mathrm{pH}$ on chemiluminescence of different probes exposed to superoxide and singlet oxygen generators. J. Biolumin. Chemilumin. 12, 277-284.

13. Sytnik, A. and I. Litvinyuk (1996) Energy transfer to a proton-transfer fluorescence probe: Tryptophan to a flavonol in human serum albumin. Proc. Natl. Acad. Sci. U S A 93, 12959-12963.

14. Wolf, F. A. D. and G. M. Brett (2000) Ligand-binding proteins: Their potential for application in systems for controlled delivery and uptake of ligands. Pharmacol. Rev. 52, 207-236.

15. Albrecht, C., J. Reichen, J. Visser, D. K. F. Meijer and W. Thormann (1997) Differentiation between naproxen, naproxen-protein conjugates, and naproxen-lysine in plasma via micellar electrokinetic capillary chromatography-A new approach in the bioanalysis of drug targeting preparations. Clin. Chem. 43, 2083-2090.

16. Guillaum, Y. C., J. Millet, L. Nicod, T. Truong-Than, C. Guinchard, A. Xicluna and M. Thomassin (2002) Solute complexation degree with human serum albumin: Biochromatographic approach. J. Chromatogr B. Analyt. Technol. Biomed. Life Sci. 768, 121-127.

17. Korinek, M., R. Dedic, A. Molnar and J. Hala (2006) The influence of human serum albumin on the photogeneration of singlet oxygen by meso-tetra(4-sulfonatophenyl)porphyrin: An infrared phosphorescence study. J. Fluoresc. (In press, DOI:10.1007/s10895-005-0057-5).

18. Takemura, T., N. Ohta, S. Nakajima and I. Sakata (1991) The mechanism of photosensitization in photodynamic therapy: Phosphorescence behavior of porphyrin derivatives in saline solution containing human serum albumin. Photochem. Photobiol. 54, 683-688.

19. Takemura, T., N. Ohta, S. Nakajima and I. Sakata (1992) The mechanism of photosensitization in photodynamic therapy: Chemiluminescence 
caused by photosensitization of porphyrins in saline containing human serum albumin. Photochem. Photobiol. 55, 137-140.

20. Silvester, J. A., G. S. Timmins and M. J. Davies (1998) Protein hydroperoxides and carbonyl groups generated by porphyrin-induced photo-oxidation of bovine serum albumin. Arch. Biochem. Biophy. 350, 249-258.

21. Wang, J., D. Xing, Y. He and X. Hu (2002) Experimental study on photodynamic diagnosis of cancer mediated by chemiluminescence probe. FEBS Lett. 523, 128-132.

22. Hao, M., D. Xing, Q. Chen and J. Wang (2004) A high sensitivity detection method of singlet oxygen and superoxide anion. Chinese Chem. Lett. 15, 679-682.

23. Lakowicz, J. R. (1999) Principles of Fluorescence Spectroscopy, Ed. 2. Kluwer Academic/Plenum publishers, New York.

24. Valensin, G., T. Kushnir and G. Navon (1982) Selective and nonselective proton spin-lattice relaxation studies of enzyme-substrate interactions. J. Magn. Reson. 46, 23.
25. Eftink, M. R. (1991) Fluorescence quenching reactions: Probing biological macro-molecular structures. In Biophysical and Biochemical Aspects of Fluorescence Spectroscopy (Edited by T. G. Dewey). Plentum Press, New York.

26. Ware, W. R. (1962) Oxygen quenching of fluorescence in solution: An experimental study of the diffusion process. J. Phys. Chem. 66, 455-458.

27. Jiang, M., M. X. Xie, D. Zheng, Y. Liu, X. Y. Li and X. Chen (2004) Spectroscopic studies on the interaction of cinnamic acid and its hydroxyl derivatives with human serum albumin. J. Mol. Struct. 692, $71-80$.

28. Akutsu, K., H. Nakajima, T. Katoh, S. Kino and K. Fujimori (1995) Chemiluminescence of Cipridina luciferin analogues. Part 2. Kinetic studies on the reaction of 2-methyl-6-phenylimidazo[1,2- $\alpha]$ pyrazin$3(7 \mathrm{H})$-one (CLA) with superoxide: Hydroperoxyl radical is an actual active species used to initiate the reaction. J. Chem. Soc. Perkin Trans. 2, 1699-1706. 